Historic, Archive Document

Do not assume content reflects current scientific knowledge, policies, or practices. 


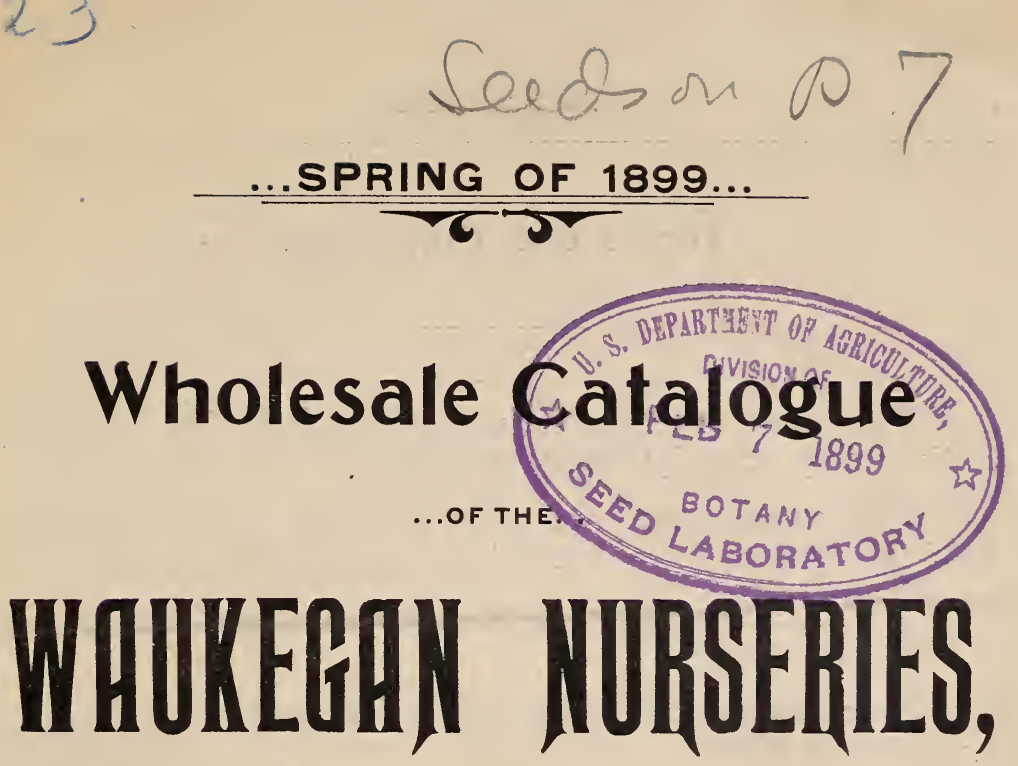

\section{WAUKEGAN, ILLINOIS.}

\section{R. DOUGLAS' SONS, proprietors.}

Our list is not a long one, as we propagate the kinds we know to be handy and valuable, and no others.

We make a specialty of growing Colorado Evergreens, which have attracted so much attention lately, both in this country and in Europe, and have by far the largest stock in cultivation.

Our Transplanted Trees are grown much further apart than is usual, allowing them room to be taken up with perfect roots.

We also make a specialty of growing Evergreen Trees from Seeds in very large quantities for Nurserymen, and Forest Trees for Planters.

We are now growing them under contract with the United States Government. 


\section{Terms and Conditions.}

The terms of payment must invariably be cash, or satisfactory security before shipping.

When goods are ordered by express, C. O. D., onehalf the amount in cash must accompany the order.

We pack in strong, light boxes, for which we make a moderate charge.

No charge is made for delivering goods at Freight or Express office.

After delivering our goods to forwarders our responsibility ceases.

When losses occur through detention or neglect, en route, claims should be make at once, on the forwarders.

Any mistake of ours will be promptly corrected if notified within ten days from receipt of goods.

Purchasers will confer a favor by stating the manner in which they wish their goods sent, by freight or express. We can ship from Waukegan by American Express; Chicago and Northwestern Railway; Elgin Joliet and Eastern Railway and from Gurnee via. United States Express and Chicago, Milwaukee and St. Paul Railway; also give usthe route from Chicago, if practicable. Write Post Office address plainly.

The articles on the following list will be furnished at annexed prices only when the quantity specified is taken except as follows: 50 at 100 rates, 500 at 1,000 rates, etc. Rates by the 100 or 1,000 , etc., will not apply when two or more articles or classes offered separately are included to make up the right number. We have by far the largest stock of Evergreens ever offered in the United States, all grown from seeds on our own grounds. 


\section{New and Rare Evergreens.}

\section{SEVERAL TIMES TRANSPLANTED.}

We have a few hundred Colorado Blue Spruce, Picea pungens, and Douglas Spruce of Colorado, 7 to 10 feet high, perfect specimens that have stood a good distance apart and are perfect in form and color, which we offer at $\$ 5.00$ and upwards per tree.

\begin{tabular}{|c|c|c|c|}
\hline & $\begin{array}{l}\text { Per } \\
\text { Pair. }\end{array}$ & Per 10. & $\begin{array}{l}\text { Per } \\
100 .\end{array}$ \\
\hline Picea pungeons, Blue form, selected..... & $\$ \begin{array}{ll}5 & 00 \\
4 & 00\end{array}$ & $\$ 2000$ & $\$$ \\
\hline $\begin{array}{lllllll}6 & 6 & 6 & 6 & 6 & \ldots \ldots 12 \text { to } 15 \text { in }\end{array}$ & 125 & $\begin{array}{rr}10 & 00\end{array}$ & 5000 \\
\hline Green form ......... 5 to $7 \mathrm{ft}$ & 400 & 1500 & \\
\hline $66 \quad 66 \quad 66 \quad \ldots \ldots$ & 150 & 1000 & \\
\hline $\begin{array}{llllllll}6 & 66 & 6 & 6 & 6 & 66 & \ldots & 2 \text { to } 3 \mathrm{ft}\end{array}$ & 100 & 400 & 3000 \\
\hline Abies Concolor, from Colorado $\underset{66}{\text { Seed }} \underset{66}{\ldots \mathrm{yrs}}$ & 150 & 500 & \\
\hline 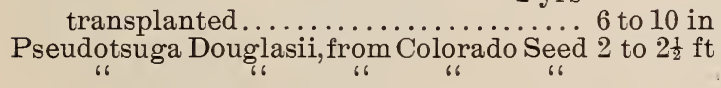 & 100 & $\begin{array}{ll}2 & 50 \\
5 & 00\end{array}$ & $\begin{array}{ll}15 & 00 \\
30 & 00\end{array}$ \\
\hline 2 years transplanted $\ldots \ldots \ldots \ldots \ldots 12$ to 15 in & & 150 & 1000 \\
\hline $\begin{array}{l}\text { Abies Siberica, Siberian silver fir...... } 2 \text { to } 3 \mathrm{ft} \\
\text { Thuya Occidentalis, Douglas Golden Arbor }\end{array}$ & 200 & 800 & \\
\hline Thuya Occidentalis, Douglas Golden Arbor & 150 & 500 & \\
\hline Thuya Occidentalis, Douglas Golden Arbor & 100 & 400 & \\
\hline Thuya Occidentalis, Douglas Pyramid 6 to 10 in & 50 & 200 & \\
\hline Arbor Vitae............................ 3 to $4 \mathrm{ft}$ & 150 & 500 & 4000 \\
\hline Thuya Occidentalis, Douglas Little Gem & 100 & 300 & 2500 \\
\hline Arbor Vitae 6 in. high, 6 in. diameter & 100 & 250 & \\
\hline Thuya Occidentalis, Siberian Arbor Vitae, $_{66}^{2 \frac{1}{2}}$ to $3 \frac{1}{2} \mathrm{ft}$ & 125 & 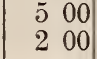 & $\begin{array}{ll}40 & 00 \\
15 & 00\end{array}$ \\
\hline
\end{tabular}

\section{Seedlings.}

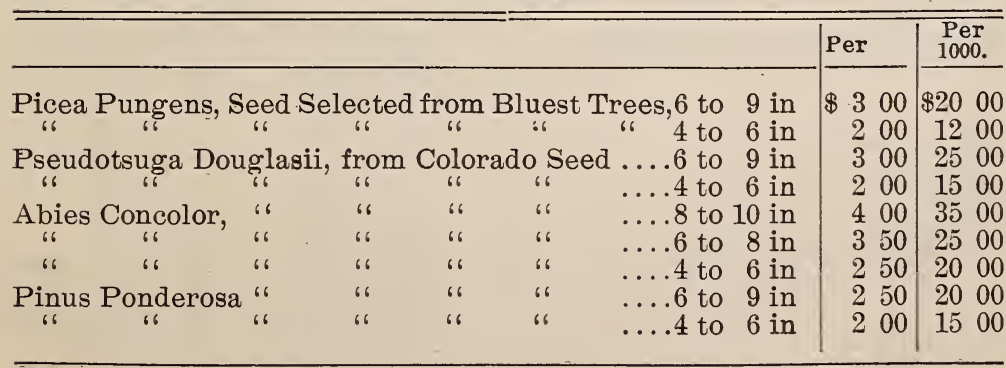




\section{Evergreens Trees}

SEVERAL TIMES TRANSPLANTED.

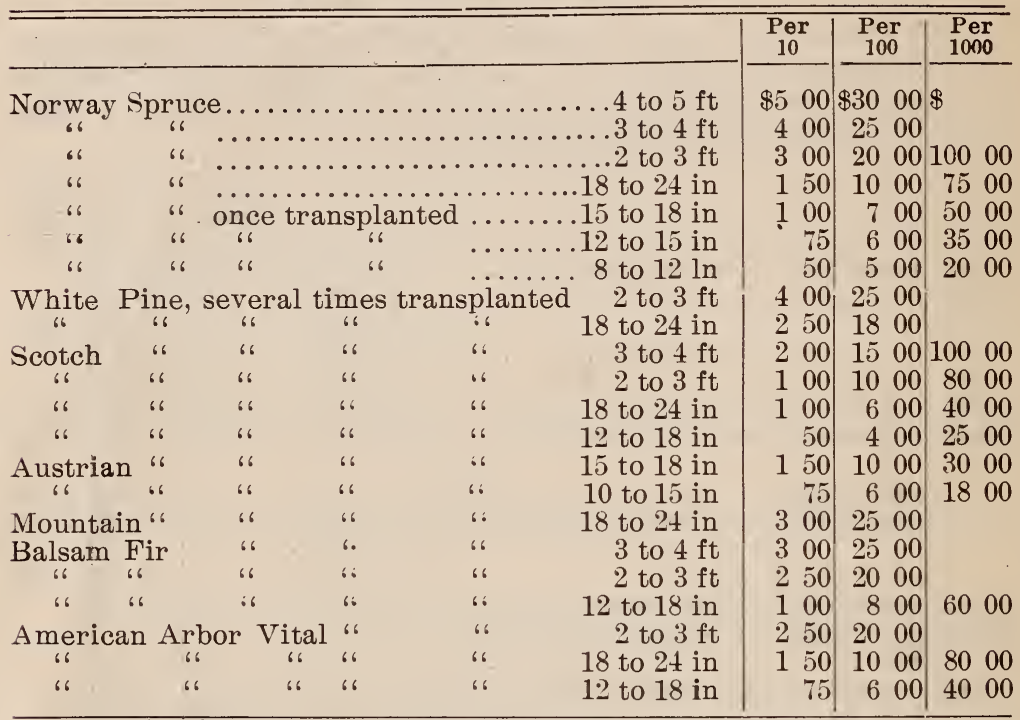

\section{Evergreen Tree Seedlings.}

The following, owing to the exceptionally fine growing season, are as fine a lot of Seedlings as we ever offered to the trade; strong, healthy and well rooted. 'They are far superior to imported Seedlings of the same age, as they make a fine growth the first season after planting. We call your especial attention to the root pruned trees. They are nearly, if not fully, equal to once transplanted trees: Next year we will have one of the largest stocks of two-year-olds we ever offered and in greater variety.

\begin{tabular}{|c|c|c|c|}
\hline & $\begin{array}{l}\text { Per } \\
100\end{array}$ & $\begin{array}{l}\text { Per } \\
1000\end{array}$ & $\begin{array}{l}\text { Per } \\
10000\end{array}$ \\
\hline Norway Spruce, root pruned.........12 to 15 in & $\$ 150$ & $\$ 1000$ & $\$ 9000$ \\
\hline 66 66 66 "6 $\ldots \ldots \ldots .10$ to 12 in & 125 & 850 & 7500 \\
\hline ........6 6 to 9 in & 100 & 750 & 6000 \\
\hline$\ldots \ldots . .4$ to 6 in & 75 & 500 & 3500 \\
\hline Scotch Pine ......... & 75 & 500 & 3500 \\
\hline Austrian $"$. . . . . . . . . & 75 & 500 & 3500 \\
\hline$\ldots \ldots \ldots \ldots \ldots \ldots$ & 100 & 700 & \\
\hline 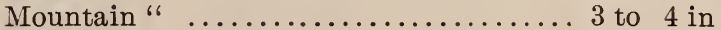 & 100 & 700 & \\
\hline American Arbor Vitae, root pruned....10 to 12 in & 125 & 1000 & 8000 \\
\hline .. 6 to 9 in & 100 & 750 & 6000 \\
\hline $66 \quad 6 \quad \ldots \ldots \ldots \ldots \ldots \ldots \ldots 4$ to & 75 & 500 & 3500 \\
\hline Balsam Fir..................... 4 to 6 in & 100 & 750 & \\
\hline
\end{tabular}




\section{Deciduous Trees Transplanted.}

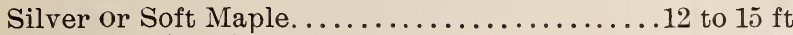

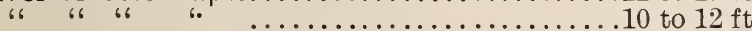
"6 " " " 6 "

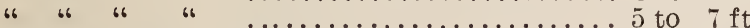

European White Birch............................... 12 tt

Tulip Tree. .......................... 8 to $10 \mathrm{ft}$

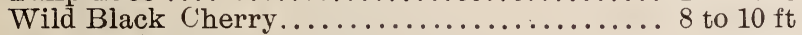

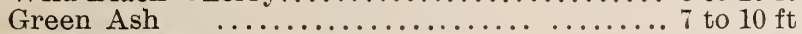

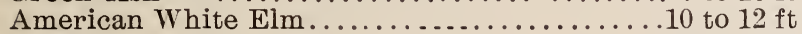

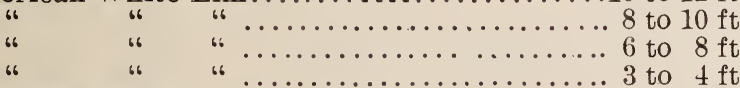

European Mountain Ash.................. to $10 \mathrm{ft}$ American " $\quad$ " $\ldots \ldots \ldots \ldots \ldots \ldots \ldots .5$ to $7 \mathrm{ft}$

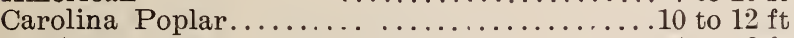

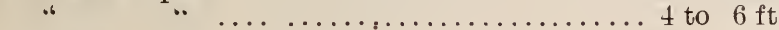
Golden $\quad$ 6 $\ldots \ldots \ldots \ldots \ldots \ldots \ldots \ldots \ldots \ldots \ldots 4$ to $5 \mathrm{ft}$ American Linden.................. 7 to $10 \mathrm{ft}$ "6 $\quad$ " $\ldots \ldots \ldots \ldots \ldots \ldots \ldots \ldots \ldots \ldots \ldots$ to $7 \mathrm{ft}$

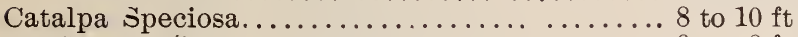
"6 "

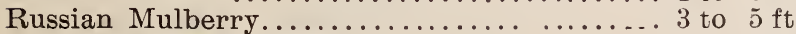
Golden Willow (Italian).................. to $5 \mathrm{ft}$

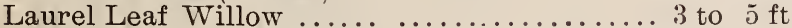
Wisconsin Weeping Willow................ 3 to $5 \mathrm{ft}$

\begin{tabular}{|cc|cc}
\hline $\begin{array}{c}\text { Per } \\
10\end{array}$ & \multicolumn{2}{c}{$\begin{array}{c}\text { Per } \\
100\end{array}$} \\
\cline { 1 - 2 }$\$ 5$ & 00 & $\$ 30$ & 00 \\
3 & 50 & 25 & 00 \\
2 & 50 & 17 & 00 \\
2 & 00 & 12 & 00 \\
6 & 00 & 50 & 00 \\
5 & 00 & 30 & 00 \\
5 & 00 & & \\
5 & 00 & 25 & 00 \\
3 & 00 & 20 & 00 \\
5 & 00 & 30 & 00 \\
3 & 50 & 25 & 00 \\
2 & 50 & 20 & 00 \\
1 & 50 & 10 & 00 \\
3 & 00 & & \\
2 & 50 & 20 & 00 \\
4 & 00 & & \\
2 & 50 & 20 & 00 \\
1 & 50 & 10 & 00 \\
1 & 50 & 15 & 00 \\
5 & 00 & 35 & 00 \\
3 & 00 & 25 & 00 \\
3 & 50 & 25 & 00 \\
2 & 50 & 20 & 00 \\
1 & 50 & 12 & 00 \\
2 & 50 & 20 & 00 \\
1 & 50 & 12 & 00 \\
1 & 50 & 12 & 00 \\
1 & 50 & 12 & 00 \\
& & & \\
& & &
\end{tabular}




\section{Forest and Ornamental Tree Seedlings.}

\begin{tabular}{|c|c|c|c|}
\hline & $\begin{array}{l}\text { Per } \\
100\end{array}$ & $\begin{array}{l}\text { Per } \\
1000\end{array}$ & $\begin{array}{r}\text { Per } \\
10000\end{array}$ \\
\hline 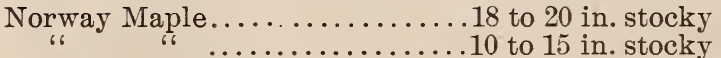 & $\$ 100$ & $\begin{array}{rr}\$ 8 & 0 \\
6 & 0\end{array}$ & $\$$ \\
\hline ilver or Soft Maple .................. 24 in & 75 & $\begin{array}{lll}4 & 0 & 0\end{array}$ & 3000 \\
\hline$\ldots \ldots \ldots 12$ to 18 in & 50 & 300 & 2500 \\
\hline$\ldots \ldots \ldots \ldots 1$ year & 75 & 500 & \\
\hline atalpa Speciosa from hom & 75 & 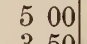 & 4000 \\
\hline 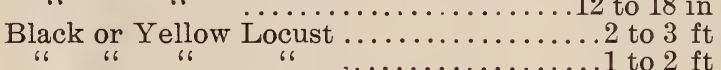 & 50 & \begin{tabular}{ll|l}
3 & 50 \\
4 & 0 & 0
\end{tabular} & 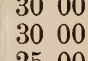 \\
\hline Green Ash or Western White $\dddot{A}_{66} \ddot{6}_{66} \ldots \ldots \ldots$....... & 50 & $\begin{array}{ll}3 & 0 \\
5 & 0 \\
\end{array}$ & 2500 \\
\hline 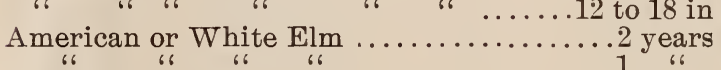 & $\begin{array}{l}50 \\
75 \\
50\end{array}$ & $\begin{array}{ll}4 & 00 \\
5 & 0 \\
3 & 5\end{array}$ & $\begin{array}{ll}30 & 00 \\
45 & 00\end{array}$ \\
\hline 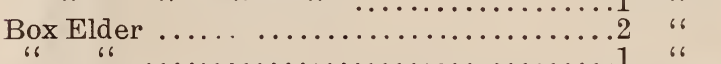 & $\begin{array}{r}50 \\
75 \\
50\end{array}$ & & \\
\hline 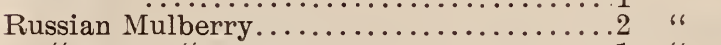 & 75 & 600 & 5000 \\
\hline $66 \quad 66$ & 50 & 400 & 350 \\
\hline White Oak .... & 100 & 800 & 700 \\
\hline Wild Black Cherry .. & 100 & 800 & 7000 \\
\hline .12 to 18 in & 75 & $\begin{array}{lll}6 & 00\end{array}$ & 500 \\
\hline .................. to 24 in & 100 & 800 & \\
\hline Ailanthus........................ 1 year & 75 & 600 & \\
\hline 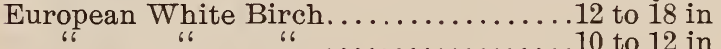 & 100 & & \\
\hline ....18 to 24 in & 100 & 800 & 7000 \\
\hline$\ldots \ldots \ldots 12$ to 18 in & 75 & 500 & 4000 \\
\hline ......6. 6 to 18 ín & 100 & 700 & 5000 \\
\hline$\nabla$ alnu & 100 & 700 & \\
\hline European Mountain Ash.............. & 75 & 500 & 4000 \\
\hline
\end{tabular}




\section{Evergreens and Deciduous Tree Seeds.}

\begin{tabular}{|c|c|c|}
\hline & Per & $\begin{array}{c}\text { Per } \\
\text { Pound. }\end{array}$ \\
\hline Abies Balsamæ, Balsam Fir.... & & $\$ 200$ \\
\hline Abies concolor, from Colorado... ............. & 80 & 400 \\
\hline Pseudotsuga Douglasii, Douglas Spruce, Colorado........ & 89 & 400 \\
\hline Picea Excelsa, Norway Spruce................. & 25 & 150 \\
\hline 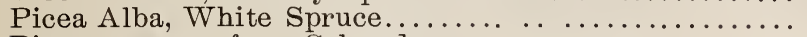 & 50 & 400 \\
\hline Picea pungens, from Colorado................ & 80 & 500 \\
\hline Pinus Austriaca, Austrian Pine.................. & 25 & 150 \\
\hline 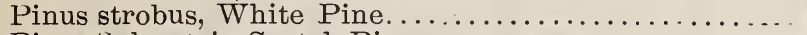 & 25 & 200 \\
\hline Pinus Sylvestris, Scotch Pine. . . . . . . . . . . . & 25 & 200 \\
\hline 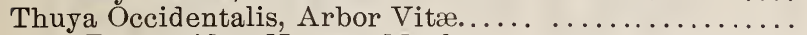 & 25 & 250 \\
\hline 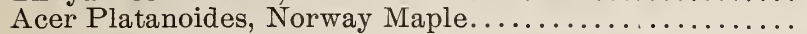 & 20 & 150 \\
\hline 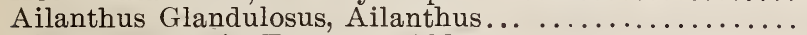 & 20 & 100 \\
\hline Alnus Communis, European Alder................. & 20 & 100 \\
\hline Betula Alba, European White Birch.............. & 25 & 150 \\
\hline Catalpa Speciosa, Hardy Catalpa............... & 25 & 150 \\
\hline Gymnocladus Canadeñsis Kentucky Coffee Tree.... & 25 & 150 \\
\hline Juglans Nigra, Black Walnut per bushel........ & & \\
\hline Juglans Cinerea, per bushel.................... 300 & & \\
\hline 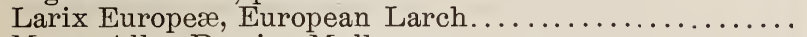 & 25 & 150 \\
\hline Morus Alba, Russian Mulberry........... & 25 & 150 \\
\hline Prunus Serotina, While Black Cherry........ & 15 & 100 \\
\hline Robinia Pseudacacia, Black Locust. ......... & 15 & 100 \\
\hline Tilia Americana, American Linden. ... . . . . . & 15 & 100 \\
\hline
\end{tabular}

We offer the following Packages by mail postpaid for $\$ 1.00$ per package.

Our mail packages are made up of the number set opposite each kind, and we never divide the packages, nor put more than one kind in each $\$ 1.00$ package.

No. $1-25$ Berberis Thunbergii,....... 2 years.

No. 2-100 Green Ash,............... 1

No. $3-100$ Hardy Catalpa, ............ 1

No. $4-100$ White Elm,..............

No. $5-100$ Wild Black Cherry............ "

No. $6-100$ Norway Spruce ............... "6

No. 7 - 50 Sweet Chestnut,............ "6

No. 8-100 American Arbor Vitae ........ 2 "

No. $9-75$ Balsam Fir, .............. "

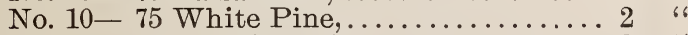

No. $11-100$ Austrian Pine................. "

No. $12-100$ Scotch Pine,................. "

No. $13-100$ Soft Maple,................. 1

No. $14-25$ Berberis Sinensis............. "

No. $15-100$ Box Elder................... "

No. 16 - 40 Douglas Spruce............... "

No. 17- 25 Picea Pungens ............. 2

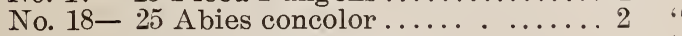

No. 19 - 50 Pinus Ponderosa .............. "

No. $20-100$ Russian Mulberry .............. " 
WE MAKE A SPECIALTY OF GROWING

\section{Hardy and Rare}
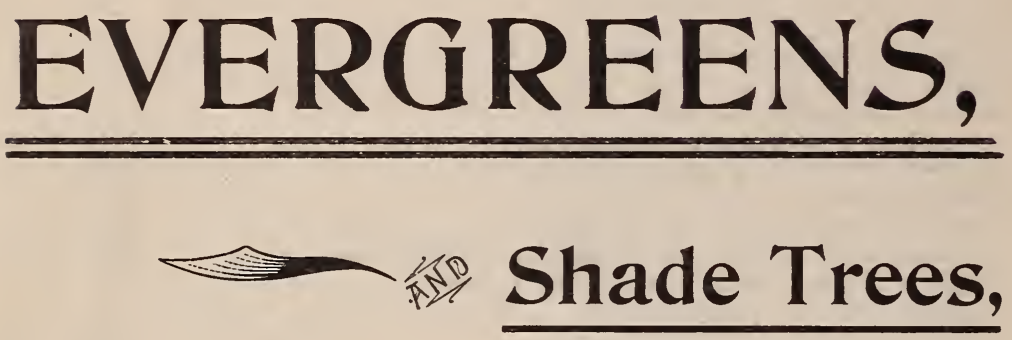

4 TO 10 FEET HIGH FOR

PARKS, CEMETERIES AND ORNATIENTAL GROUNDS.

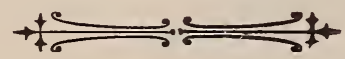

And as two of our. Nurseries must be vacated next Spring, we offer the above at special reduced rates in carloads. 